\title{
Communautés de sous-bois des peupleraies artificielles : relation entre phytomasse, richesse spécifique et perturbations
}

\author{
Marc Laquerbe \\ Centre d'écologie des systèmes aquatiques continentaux, Cnrs (UMR C 5576) 29, rue Jeanne Marvig, \\ BP 4349,31055 Toulouse cedex, France
}

(Reçu le 23 octobre 1998 ; accepté le 25 mars 1999)

\begin{abstract}
Understory plant communities of cultivated polar groves: relationship between phytomass, species richness and disturbance. Understory plant communities of cultivated poplar groves are submitted to strong disturbances essentially due to mechanical upkeep. Upkeep consists of "disking", i.e. weeding by a metallic disk which totally destroys and buries vegetation. This upkeep differs among poplar groves: the intensity decreases with tree age and it totally disappears during the years preceding clearing. With canopy closure, these differences lead to a modification in species communities. The four poplar groves chosen for this study showed different upkeep levels. Phytomass and species richness were studied by sampling vegetation at its stage of maximal growth, just before upkeep periods. As the intensity of upkeep treatment decreases with time, communities exhibited different successional stages, from annual pioneer, meadow perennial and woodland ligneous species. The results show that species richness increases with upkeep intensity, whereas phytomass values decrease. Most of these phytomasses were between 250 and $1000 \mathrm{~g} \mathrm{~m}^{-2}$ with a maximum at $2000 \mathrm{~g} \mathrm{~m}^{-2}$ in the oldest and less maintained poplar groves. For strong disturbance levels, species richness was high and reached a maximum for moderate phytomasses. However, the steady state existing in abandoned poplar groves exhibited strong phytomass accumulation near trees. On the one hand, community species enrichment mainly depended on disturbance levels induced by upkeep. On the other hand, the flora enrichment was due to maturation of plantation. We can observe an increase of species, characteristic of a more stable site. This phenomenon exists only in the oldest plantations. (C) 1999 Éditions scientifiques et médicales Elsevier SAS.
\end{abstract}

species richness / phytomass / diversity / vegetal community / disturbance / artificial poplar grove

Résumé - Les communautés de sous-bois des peupleraies artificielles sont soumises à des perturbations importantes liées à des entretiens mécaniques. Ces derniers diffèrent selon les peupleraies : ils diminuent avec l'âge des arbres pour totalement disparaître dans les dernières années avant l'abattage de la plantation. Ce changement entraîne, en liaison avec la fermeture du couvert, une modification de la couverture végétale herbacée et ligneuse du sous-bois. La phytomasse et la richesse spécifique sont étudiées par récolte de la végétation, à son maximum de développement, avant les périodes d'entretien.

En relation avec la diminution de l'entretien on peut observer l'apparition progressive d'espèces annuelles pionnières, puis de prairiales pérennes et enfin d'espèces sylvatiques, dans la majorité des cas ligneuses. Les valeurs de phytomasses sont comprises entre 250 et $1000 \mathrm{~g} \mathrm{~m}^{-2}$ avec un maximum de $2000 \mathrm{~g} \mathrm{~m}^{-2}$ dans les peupleraies les plus âgées et les moins entretenues. Dans le cas de fortes perturbations, la richesse spécifique est élevée et atteint un maximum pour des valeurs modérées de phytomasse. Dans les peupleraies abandonnées on observe une forte accumulation de phytomasse près des arbres. L'augmentation du nombre d'espèces dépend principalement des niveaux de perturbation causés par l'entretien. Cependant les résultats obtenus laissent supposer que l'enrichissement de la flore par maturation permet l'apparition d'espèces de milieux plus stables, à durée de vie plus élevée. Ce phénomène ne s'observe que dans les plantations les plus âgées. (-) 1999 Éditions scientifiques et médicales Elsevier SAS.

richesse spécifique / phytomasse / diversité / communauté végétale / perturbation / peupleraie artificielle

* Correspondence and reprints

laquerbe @ cesac.cemes.fr 


\section{Introduction}

De nombreux mécanismes et modèles ont été proposés pour expliquer le nombre d'espèces qui peuvent coexister dans un écosystème. De faibles perturbations [1], des taux de nutriments élevés [2] ou la stabilité de l'écosystème [3] peuvent contribuer à l'augmenter.

Bien que l'on admette une relation entre la productivité (la phytomasse, la biomasse ou la production) et la diversité (ou la richesse spécifique) $[4,5]$, on ne peut réellement l'expliquer. Le maximum de richesse spécifique correspond généralement à des taux intermédiaires de biomasse $[6,7]$. Les hypothèses avancées jusqu'à présent restent théoriques et contradictoires [8]. Les résultats ayant trait à cette mise en relation sont différents suivant les auteurs : si la majorité d'entre eux indiquent une relation unimodale entre les deux facteurs considérés, quelques uns évoquent une relation linéaire [9].

Le cas étudié ici est celui de peupleraies plantées. Elles sont fréquentes en moyenne vallée de la Garonne [10], et se situent principalement dans la plaine alluviale. Bénéficiant d'une bonne humidité du sol ainsi que de fortes concentrations en nutriments [11], le sous-bois est envahi par une végétation luxuriante. Mais le peuplier, exploité pour le bois, est très sensible à la concurrence végétale [12]. Des entretiens mécaniques sont donc réalisés pour maîtriser le développement de la végétation spontanée.

Même si les peupleraies n'ont pas pour but de favoriser la diversité des espèces végétales herbacées mais de produire du bois [13], le maintien de la diversité spécifique est récemment pris en compte dans les aménagements forestiers [14, 15].

L'objectif de ce travail est de préciser le rôle et l'impact de ce type de perturbation sur les communautés de sous-bois et de rechercher les relations pouvant exister entre la richesse spécifique et la phytomasse. Cette mise en relation prendra compte des modalités d'entretien et des conditions environnementales qui différencient les peupleraies étudiées.

\section{Matériels et méthodes}

\subsection{Site d'étude}

Les peupleraies étudiées se situent dans la plaine alluviale de la moyenne vallée de la Garonne, à une quarantaine de kilomètres au nord de Toulouse, près de Verdun-sur-Garonne (Tarn et Garonne, France). Situées en continuité les unes des autres, ces peupleraies sont plantées sur des substrats de texture limono-argileuse en surface et sablo-limono-argileuse au-dessous de soixante centimètres. Le $\mathrm{pH}$ varie de 7.4 à 8.5 .

Près du fleuve, la végétation naturelle est représentée par une saulaie-peupleraie à Salix alba et Populus nigra et, au sein des terres, à une frênaie-ormaie (Fraxinus angustifolia subsp. oxycarpa, Ulmus minor). Les terrasses sont peuplées de chênaies (Quercus pubescens et Quercus robur).

\subsection{Caractères des peupleraies}

Le travail porte sur quatre peupleraies, nommées respectivement $\mathrm{P} 1, \mathrm{P} 2$, $\mathrm{P} 3$ et $\mathrm{P} 4$. Elles décrivent l'évolution théorique de peupleraies plantées : d'une jeune exploitation, très entretenue qui présente un couvert forestier très ouvert, à des plantations plus âgées, dont le couvert se ferme peu à peu. Ces dernières perçoivent un entretien de plus en plus réduit voir absent les dernières années avant l'abattage.

En plaine alluviale, c'est le disquage qui est le plus couramment employé. Grâce à une charrue à disques, il $y$ a destruction totale et enfouissement sur place de la végétation du sous-bois.

La peupleraie P1 est âgée de 4 ans. Son disquage est croisé : les disques passent entre les arbres, dans deux directions perpendiculaires. La peupleraie $\mathrm{P} 2$, plus âgée (13-15 ans), présente un couvert forestier plus dense. L'entretien est limité à deux passages par an. P3 et P4 ne sont pas soumises à des interventions depuis quatre ans, mais P4 est beaucoup plus âgée ( 35 ans) que P3 (18 ans). La majorité des peupleraies est plantée en $I$ 45-51, sauf P4 en Robusta. Ce dernier est un clone ancien, à long cycle de croissance, ce qui explique l'âge de la peupleraie.

\section{3. Échantillonnage}

En relation avec le passage du disque d'entretien et l'emplacement des arbres, nous avons défini trois localisations de prélèvement. Dans les peupleraies étudiées, nous entendons par localisation la position relative de chaque observation. Appelées L1, L2 et L3, elles sont respectivement situées : autour d'un peuplier, entre deux peupliers et au centre de quatre arbres. Leur emplacement au sein de la peupleraie correspond à différentes intensités de perturbation (figure l).

La végétation est prélevée dans chacune des localisations L1, L2 et L3. Chacune d'elles est étudiée à l'aide de trois répétitions. Chaque répétition correspond à un quadrat d' $1 \mathrm{~m}^{2}$ de surface $(1 \times 1 \mathrm{~m})$. Leur emplacement est défini par un traitement informatique utilisant un système 


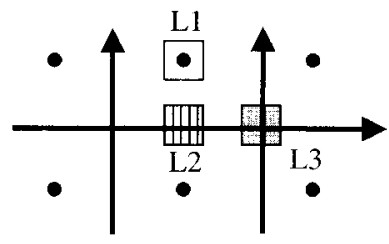

Entretien croisé

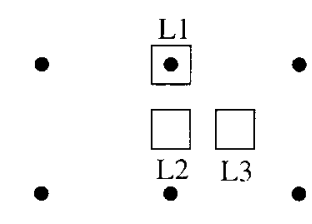

Abandon
Figure 1. Modalités d'entretien des peupleraies et localisations d'observation.

Perturbation locale liée à l'entretien : 때, absence de perturbation ; $\square$ perturbation moyenne ;

perturbation forte.

de tirage au hasard (Méthode Monte-Carlo). Le tirage a été réalisé à partir d'une grille indiquant les différentes localisations possibles au sein de chaque peupleraie,

Cette opération a été effectuée en mai-juin 1995, au maximum de développement de la végétation, avant les premiers entretiens. Le prélèvement de la végétation est opéré par coupe manuelle au niveau du collet. A chaque prélèvement, nous mesurons la richesse et la composition spécifique, par comptage et détermination des espèces présentes. Les plantes vasculaires, nommées selon [16], et les bryophytes, selon [17], sont prises en compte. Par la suite, les espèces collectées sont placées dans une étuve à $105^{\circ} \mathrm{C}$ et séchées jusqu'à poids constant. Après pesage, nous déterminons la phytomasse, regroupant biomasse et nécromasse (sur pied et détachée).

Sur chacun des quadrats récoltés, nous avons établi une classification selon le cycle de vie des espèces (annuelles, bisannuelles et vivaces). Nous avons comptabilisé les mousses, les espèces ligneuses et non ligneuses. Nous avons signalé la part des espèces qui développent un système traçant (rhizomes ou stolons).

A l'aide des résultats obtenus, nous calculons la diversité $(\mathrm{H})$ à l'aide de l'indice de Shannon Wiener [18] : - $\Sigma$ $\mathrm{p}_{\mathrm{i}} \log _{2} \mathrm{p}_{\mathrm{i}}$, avec $\mathrm{p}_{\mathrm{i}}$, rapport entre le poids de l'espèce $\mathrm{i}$ et le poids total du relevé ; ainsi que l'équitabilité (J) selon
[19] : $\mathrm{J}=\mathrm{H} / \log _{2}(n)$, avec $\log _{2}(n)$ diversité maximale pour un nombre $n$ d'espèces.

\subsection{Analyses statistiques}

Afin de déterminer les différences observées dans les caractéristiques des communautés végétales des points d'observation (localisations) d'une même peupleraie, nous avons utilisé un test de Tukey [20]. Les données obtenues ont subi une transformation logarithmique afin de les normaliser. Les analyses ont été réalisées avec Systat [21].

\section{Résultats}

\subsection{Caractéristiques de la végétation}

À l'échelle de la peupleraie, le disquage favorise la richesse spécifique, la diversité et l'équitabilité des communautés végétales de sous-bois et diminue la phytomasse (tableau I).

Les localisations L1, non perturbées par le passage de la charrue, présentent une richesse spécifique d'autant plus élevée que la peupleraie est entretenue, le maximum étant atteint pour la peupleraie P1 (figure 2). Dans les peupleraies entretenues $\mathrm{P} 1$ et $\mathrm{P} 2$, les localisations L2 montrent une richesse spécifique significativement plus élevée qu'en $\mathrm{L} 1(P<0,05$ pour $\mathrm{P} 1$ et $P<0,01$ pour $\mathrm{P} 2$, tableau II). Lors de l'exposition maximale à l'entretien, cas de la localisation L3, il y a augmentation de richesse spécifique en P2. En P1 cette localisation montre d'une part une perturbation forte, entretien croisé, et une fréquence de passage très élevée, trois passages par an (cf. figure 1). Le nombre d'espèces enregistré est significativement $(P<0,05)$ plus faible qu'en L2. Pour les peupleraies abandonnées, le nombre d'espèces des localisations L2 et L3 est légèrement inférieur à celui de la localisation L1, mais sans différence significative.

En ce qui concerne la phytomasse, les résultats indiquent qu'elle est fonction inverse du degré d'entretien

Tableau I. Richesse spécifique, phytomasse, diversité et équitabilité des communautés de sous-bois selon la peupleraie (moyenne \pm intervalle de confiance à $95 \%$ ).

\begin{tabular}{lcccc}
\hline & P1 & P2 & P3 & P4 \\
\hline Richesse spécifique (nb. esp./m ${ }^{-2}$ ) & $24.8 \pm 3.6$ & $12.9 \pm 3$ & $5.1 \pm 0.7$ & $8.6 \pm 1.6$ \\
Phytomasse $\left(\mathrm{g} . / \mathrm{m}^{-2}\right.$ ) & $418.4 \pm 61$ & $559.7 \pm 101.5$ & $798 \pm 418.8$ & $1036.4 \pm 576.5$ \\
Diversité (bits) & $3.1 \pm 0.3$ & $1.9 \pm 0.3$ & $0.9 \pm 0.6$ & $1.4 \pm 0.3$ \\
Equitabilité & $0.7 \pm 0.05$ & $0.5 \pm 0.07$ & $0.3 \pm 0.1$ & $0.4 \pm 0.1$ \\
\hline
\end{tabular}



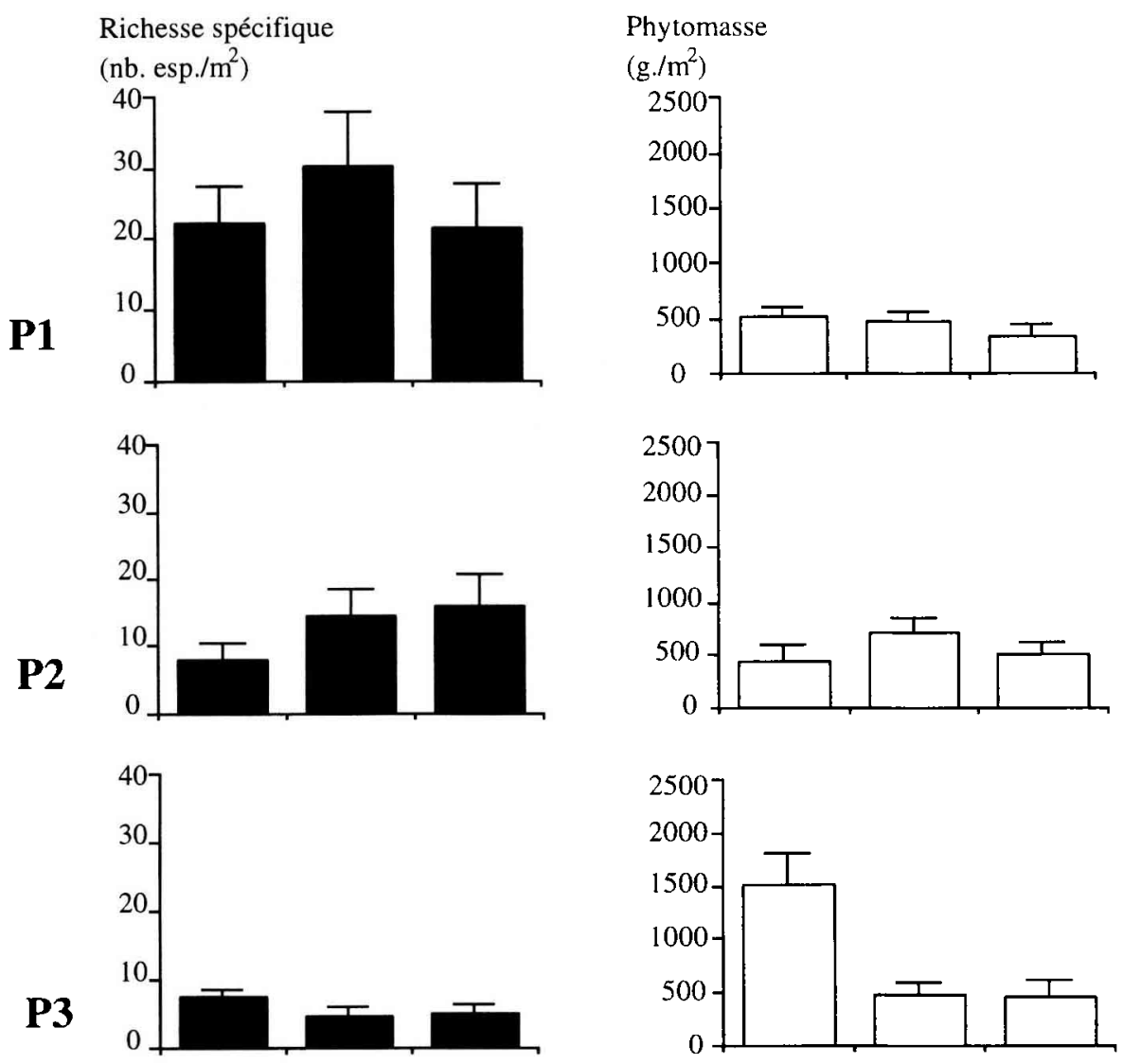

P3
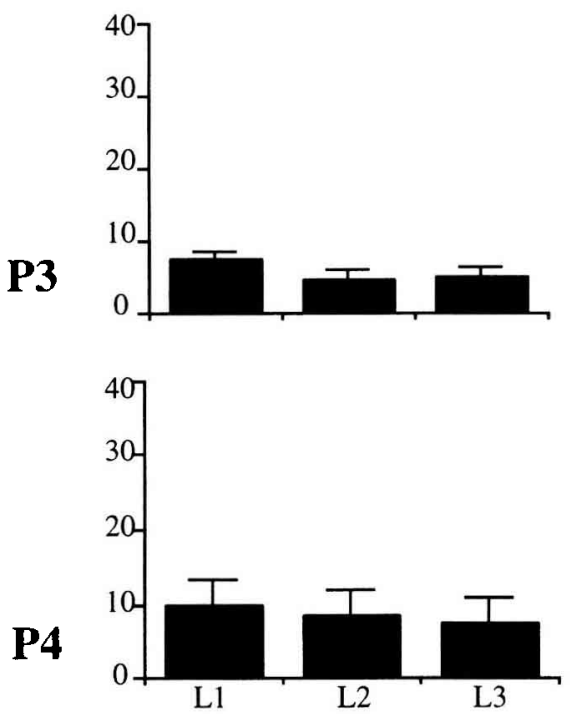

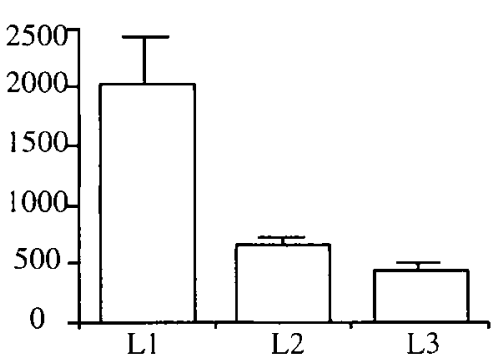

Figure 2. Richesses spécifiques et phytomasses (moyenne \pm intervalle de confiance à $95 \%$ ) selon la peupleraie et la localisation d'observation. avec, de L1 à L3, une diminution des valeurs de phytomasse en fonction de l'intensité et de la fréquence d'entretien (figure 2). Ces différences ne sont significatives qu'entre les localisations L2 et L3 de P1, L1-L2 et L2-L3 de P2 (tableau II). Dans les peupleraies abandonnées ( $\mathrm{P} 3$ et $\mathrm{P} 4$ ) la localisation $\mathrm{L} 1$ développe une importante végétation, composée essentiellement de ligneux. Les valeurs obtenues, bien que variables, sont très significativement différentes $(P<0,001)$ de celles obtenues au sein des localisations L2 et L3.
Les espèces observées sont principalement des espèces mésophiles (Bromus sterilis, Lapsana communis) à mésohygrophiles (Arhenatherum elatius, Galium aparine, Rubus caesius, Urtica dioica) (tableau III). Ces dernières sont pour la plupart exigeantes en azote.

Le vieillissement d'une exploitation populicole est matérialisé de P1 à P4. L'étude de leur sous-bois montre le passage des pionnières annuelles en $\mathrm{P} 1$ (Veronica persica, Valerianella locusta, Cerastium glomeratum...) à 
Tableau II. Effet de la localisation du prélèvement sur les valeurs de richesse spécifique et de phytomasse. Moyenne des erreurs standards et probabilités associées selon un test de Tukey.

\begin{tabular}{lccc}
\hline Peupleraie & Localisation & $\begin{array}{c}\text { Richesse } \\
\text { spécifique }\end{array}$ & Phytomasse \\
\hline P1 & L1L2 & $0.13^{*}$ & $\mathrm{~ns}$ \\
& L1L3 & $\mathrm{ns}$ & $\mathrm{ns}$ \\
& L2L3 & $-0.15^{*}$ & $-0.15^{*}$ \\
P2 & L1L2 & $0.26^{* *}$ & $0.21^{* *}$ \\
& L1L3 & $0.3^{* *}$ & $\mathrm{~ns}$ \\
& L2L3 & $\mathrm{ns}$ & $-0.14^{*}$ \\
P3 & L1L2 & $\mathrm{ns}$ & $-0.51^{* * *}$ \\
& L1L3 & $\mathrm{ns}$ & $-0.57^{* * *}$ \\
& L2L3 & $\mathrm{ns}$ & $\mathrm{ns}$ \\
& L1L2 & $\mathrm{ns}$ & $-0.49 * * *$ \\
P4 & L1L3 & $\mathrm{ns}$ & $-0.66^{* * *}$ \\
& L2L3 & $\mathrm{ns}$ & $-0.17^{* *}$ \\
& & & \\
\hline
\end{tabular}

Note : ns, non significatif ; ${ }^{*}, \mathrm{p}<0.05 ;{ }^{* *}, \mathrm{p}<0.01 ; * * *, \mathrm{p}<0.001$.

des espèces prairiales perennes en P2 (Arrhenatherum elatius, Agrostis stolonifera...) puis sylvatiques et ligneuses (Cornus sanguinea, Quercus robur, Ulmus minor...) en P3 et P4. Suivant ce gradient, la dominance des espèces devient de plus en plus importante.

Dans la peupleraie la plus fortement et fréquemment soumise à l'entretien, $\mathrm{P} 1$, les trois types biologiques annuel, bisannuel et vivace coexistent (tableau $I V$ ). Dans les peupleraies entretenues $\mathrm{P} 1$ et $\mathrm{P} 2$, la part prise par les espèces annuelles est d'autant plus importante que la localisation est soumise à un entretien important : de L1 à L3. Les peupleraies dépourvues d'entretien P3 et P4 présentent majoritairement des espèces vivaces. Seules les localisations $\mathrm{L} 2$ et $\mathrm{L} 3$ récemment abandonnées ont légèrement plus d'espèces annuelles que la localisation du pied de l'arbre, L1.

Le pourcentage des mousses, compris entre 0 et $13 \%$, reste relativement faible. Elles sont surtout présentes dans les peupleraies les moins entretenues, P3 et P4. Dans les peupleraies entretenues, $\mathrm{P} 1$ et $\mathrm{P} 2$, les mousses sont localisées en Ll, zone la moins perturbée de la peupleraie,

Les espèces non ligneuses constituent la majeure partie des communautés rencontrées. Seules les peupleraies P3 et P4, dépourvues d'entretien, montrent des taux élevés de ligneux, essentiellement au pied de l'arbre (L1).

Les espèces qui développent un système traçant sont fortement représentées dans l'ensemble des peupleraies étudiées. Seule P1, fortement entretenue, présente une réduction de ces espèces. Au sein des peupleraies $\mathrm{Pl}$ et $\mathrm{P} 2$, on observe de L1 à L3, en relation avec l'exposition à l'entretien, une diminution du pourcentage des espèces traçantes. Dans les peupleraies abandonnées, P3 et P4, ces espèces deviennent majoritaires représentant ainsi 40 à $70 \%$ des espèces.

\subsection{Relation entre phytomasse et richesse spécifique}

Au cours de la période de récolte, la plupart des valeurs de phytomasse sont comprises entre 250 et $1000 \mathrm{~g} \mathrm{~m}^{-2}$ (figure 3). On y trouve les valeurs maximales de richesse spécifique. Il n'existe pas de phytomasses inférieures à $250 \mathrm{~g} \mathrm{~m}^{-2}$. Lorsque les valeurs sont supérieures à $1000 \mathrm{~g} \mathrm{~m}^{-2}$, la richesse spécifique est faible. C'est le cas de certaines localisations des peupleraies $\mathrm{P} 3$ et $\mathrm{P} 4$. Seules les localisations $\mathrm{L} 1$ de $\mathrm{P} 3$ et $\mathrm{P} 4$, qui n'ont jamais été entretenues depuis le moment de la plantation, présentent des valeurs élevées de phytomasse.

Tableau III. Phytomasse relative (rapport poids sec de l'espèce sur poids total de l'échantillon) des dix espèces les plus répandues dans les sous-bois des peupleraies étudiées.

\begin{tabular}{|c|c|c|c|c|c|c|c|c|}
\hline Rang & Espèces & $\begin{array}{c}\text { Phyt. Re } \\
(\%)\end{array}$ & Espèces & $\begin{array}{c}\text { Phyt. Rel. } \\
(\%)\end{array}$ & Espèces & $\begin{array}{c}\text { Phyt. Re } \\
(\%)\end{array}$ & Espèces & $\begin{array}{c}\text { Phyt. Rel. } \\
\text { (\%) }\end{array}$ \\
\hline 1 & Poa trivialis & 14.3 & Arrhenatherum elatius & 35 & Rubus caesius & 41 & Cornus sanguinea & 54.6 \\
\hline 3 & Rubus caesius & 10.6 & Bromus sterilis & 11.2 & Rubus ulmifolius & 12.5 & Galium aparine & 8.5 \\
\hline 4 & Veronica persica & 8.8 & Rubus caesius & 8.8 & Equisetum telmateia & 2.5 & Hedera helix & 2.7 \\
\hline 5 & Galium aparine & 6.1 & Galium aparine & 7.1 & Circaea lutetiana & 2.5 & Sambucus ebulus & 1.7 \\
\hline 8 & Elymus repens & 4.5 & Eupatorium cannabinum & $n 2.9$ & Homalothecium sericeum & $0.2 \mathrm{~L}$ & Eupatorium cannabinum & im 0.7 \\
\hline 9 & Torilis arvensis & 4.5 & Geranium dissectum & 1.9 & Clematis vitalba & 0.2 & Angelica sylvestris & 0.7 \\
\hline 10 & Sonchus oleraceus & 3.6 & Bromus hordeaceus & 1.8 & Hedera helix & 0.1 & Ulmus minor & 0.7 \\
\hline
\end{tabular}




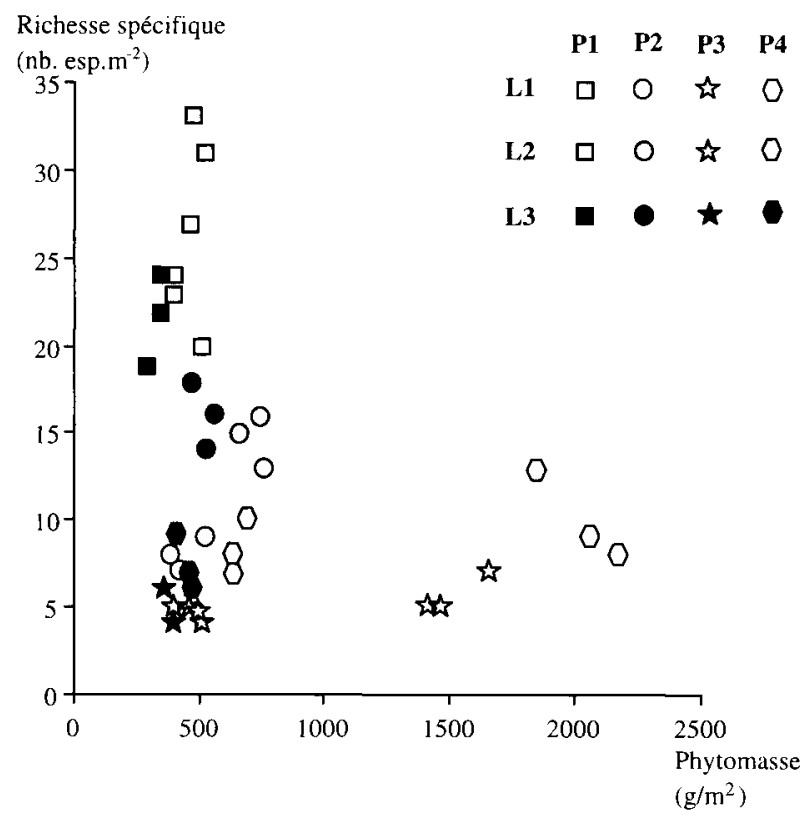

Figure 3. Mise en relation de la phytomasse et de la richesse spécifique des communautés de sous-bois, selon la peupleraie et la localisation d'observation.

Ce n'est pas le cas de L2 et L3, récemment dépourvues d'entretien.

Entre 250 et $1000 \mathrm{~g} \mathrm{~m}^{-2}$ les valeurs de richesse spécifique, réparties sur l'axe $Y$, sont d'autant plus élevées que la perturbation est importante, avec progressivement ordination de : localisations L2 et L3 de P3 et P4 (non entretenues), des valeurs enregistrées en P2 (deux disquages par an) et celles obtenues en P1 (trois disquages par an). A des valeurs supérieures de phytomasse, la richesse spécifique enregistrée se stabilise autour de 5 esp. $\mathrm{m}^{-2}$. Seules les localisations L1 de P4 montrent un nombre plus élevé d'espèces. Ces valeurs sont comparables à celles enregistrées dans la peupleraie $\mathbf{P} 2$.

\section{Discussion}

La peupleraie artificielle représente un compromis entre un milieu riche en nutriments, lié à plaine alluviale, qui facilite l'installation d'un grand nombre d'espèces [22], et un entretien régulier, qui détruit la végétation en place ouvrant ainsi de nouvelles zones de colonisation. La destruction du sous-bois se réalise sans affecter le couvert forestier. Les écosystèmes qui présentent une forte productivité sont conduits vers à un déclin de l'hétérogénéité spatiale [23] et à la dominance de quelques espèces [7]. Dans notre cas, l'entretien permet d'y remédier et favorise la biodiversité.

Huston [24] a mis en place un modèle qui tient compte de la périodicité des perturbations, ainsi que des phénomènes de compétition. Il observe un maximum de diversité à des niveaux intermédiaires de fréquence de perturbation et de taux de productivité. Il existe un contraste entre les espèces capables de résister à de fortes perturbations et les espèces compétitrices, qui ont besoin de conditions stables pour s'établir et croître. Seules les annuelles sont parfaitement capables de s'adapter aux perturbations [25] en élaborant d'importantes banques de graines leur assurant une colonisation rapide de milieux ouverts [26]. Dans les peupleraies, on trouve une relation directe entre le niveau de perturbation et la part prise par les annuelles. Raunkiaer [27] classe ces espèces comme des thérophytes. Par adaptation à des perturbations répétées, on parle d'espèces rudérales sensu Grime [7]. En peupleraie entretenue, les espèces annuelles se répartissent suivant le degré d'exposition à l'entretien du sol, de L1 à L3. Leur implantation est ainsi défavorisée au pied des arbres [28].

Tableau IV. Pourcentage d'espèces annuelles, bisannuelles et vivaces ; de mousses, non ligneux et ligneux ainsi que d'espèces traçantes selon la peupleraie et la localisation d'observation.

\begin{tabular}{|c|c|c|c|c|c|c|c|c|c|c|c|c|}
\hline & \multicolumn{3}{|c|}{ P1 } & \multicolumn{3}{|c|}{ P2 } & \multicolumn{3}{|c|}{ P3 } & \multicolumn{3}{|c|}{ P4 } \\
\hline & L1 & L2 & L3 & L1 & L2 & L3 & L1 & $\mathrm{L} 2$ & L3 & $\mathrm{L} 1$ & L2 & L3 \\
\hline Annuelles (\%) & 38.9 & 53.5 & 63.6 & 15.4 & 36.8 & 47.6 & 10 & 22.2 & 12.5 & 6.7 & 13.3 & 8.3 \\
\hline Bisannuelles (\%) & 5.6 & 7 & 3.1 & 0 & 0 & 0 & 0 & 0 & 0 & 0 & 0 & 0 \\
\hline Vivaces $(\%)$ & 55.5 & 39.5 & 33.3 & 84.6 & 63.2 & 52.4 & 90 & 77.8 & 87.5 & 93.3 & 86.7 & 91.7 \\
\hline Mousses (\%) & 2.7 & 0 & 0 & 2.9 & 0 & 0 & 10 & 0 & 12.5 & 13.3 & 6.7 & 8.3 \\
\hline Non ligneux $(\%)$ & 94.6 & 97.7 & 97.1 & 90 & 89.5 & 100 & 40 & 55.6 & 62.5 & 60 & 80 & 75 \\
\hline Ligneux (\%) & 2.7 & 2.3 & 2.9 & 7.1 & 10.5 & 0 & 50 & 44.4 & 25 & 26.7 & 13.3 & 16.7 \\
\hline Espèces traçantes (\%) & 29.7 & 25.6 & 17.6 & 69.2 & 47.4 & 42.1 & 60 & 66.7 & 62.7 & 46.7 & 46.7 & 33.3 \\
\hline
\end{tabular}


Par baisse du régime d'entretien on voit apparaître des espèces traçantes (Agrostis capillaris, Agrostis stolonifera, Arhenatherum elatius, Cynodon dactylon...) qui colonisent le milieu par de nombreux rejets. Dans les conditions les plus stables, les ligneux sont prépondérants. Les mousses sont, quant à elles, faiblement représentées, ce qui avait déjà été signalé par Botineau [29]. Un substrat trop souvent renouvelé en limite la dispersion et la croissance [30]. Avec l'âge de la peupleraie le couvert forestier se ferme peu à peu, l'entretien est réduit et finit par disparaître, enfin l'ensemble perçoit un vieillissement général menant à une certaine stabilité. On passe d'un espace pionnier, créé et maintenu par une destruction de la couverture végétale ainsi qu'un couvert peu dense, à un espace semi-naturel qui reflète l'équilibre entre l'histoire et les conditions environnementales de la plantation.

Nos données indiquent qu'à des productions modérées de phytomasse (250-1000 $\mathrm{g} \mathrm{m}^{-2}$ ), la richesse spécifique est d'autant plus élevée que le degré de perturbation est élevé. Au-delà, la richesse spécifique prend des valeurs inférieures. Cette tendance est généralement celle que l'on trouve dans les systèmes de plantes herbacées $[2,7]$. Grime [7] indique que le stress, la perturbation et la dominance contrôlent la richesse spécifique. Wilsson et Keddy [31] ajoutent que le degré de compétition varie suivant le type de végétation en fonction de la phytomasse qu'il développe. C'est ce qu'on observe lorsqu'il y a arrêt des entretiens de la peupleraie ; où les espèces, initialement herbacées, cèdent la place à des ligneux. La quantité de lumière, de moins en moins disponible au fur et à mesure du vieillissement d'une peupleraie, joue un rôle important dans l'établissement et la croissance des espèces végétales [32]. En raison des résultats obtenus en P1, ce modèle n'est pas en liaison avec les observations d'Odum [33] qui indiquent que la plus forte diversité en espèces s'observe à des taux modérés de gradients physiques. La peupleraie P1 offre, tout au long de l'année, une faible couverture forestière. Elle constitue un cas différent des autres peupleraies. Mais de ce fait, elle peut présenter des conditions favorables supplémentaires où les niveaux de perturbation plus élevés sont facteurs d'enrichissement et non d'élimination spécifique. Selon Valverde et Silvertown [34], les premiers stades de fermeture de la canopée ont les effets les plus déterminants sur les caractéristiques des communautés de sous-bois. Dans les peupleraies où le couvert est plus dense, la richesse spécifique montre des valeurs élevées dans la peupleraie entretenue, $\mathrm{P} 2$. Nous ne savons pas si, dans ce cas, une intensification de l'entretien faciliterait ou diminuerait la richesse spécifique.

L'accumulation de litière des arbres, conséquence de l'absence d'entretien en P3 et P4, engendre des contraintes physiques qui peuvent faire obstacle à l'émergence et à la régénération des espèces [35]. Seule les localisations $\mathrm{L} 1$, des peupleraies $\mathrm{P} 3$ et $\mathrm{P} 4$, n'ont jamais été entretenues. On y remarque donc le développement de ligneux arbustifs et de fortes accumulations de phytomasse. Les résultats obtenus dans la peupleraie $\mathrm{P} 4$ montrent que les phénomènes de stabilisation à long terme du milieu engendrent un nombre plus élevé d'espèces. Cette tendance n'est visible qu'en localisation L1, pied de l'arbre, mais pourrait, sur des échelles de temps plus longues, se généraliser à l'ensemble de la peupleraie, Ceci rejoint l'idée selon laquelle les écosystèmes les plus stables sont les plus diversifiés [36]. Cette relation est reconnue comme un dogme écologique en dépits de résultats contradictoires $[37,38]$.

On peut généraliser les phénomènes observés de la façon suivante.

L'entretien favorise la richesse spécifique. En relation avec un couvert forestier peu dense, une litière peu abondante et un sous-bois jeune, la richesse spécifique atteint les plus fortes valeurs (cas de P1). Le substrat présente la propriété de ne pas être limité en nutriments, aussi les valeurs de phytomasse sont élevées. Dans les peupleraies entretenues, ces valeurs sont peu différentes les unes des autres. Il est donc difficile à mettre en évidence des tendances bien marquées. Tilman et Pacala [39] parlent d'un pic de diversité à des niveaux intermédiaires de nutriments, ce qui n'a pas pu être vérifié ici.

L'enrichissement spécifique dû à l'entretien est un phénomène éphémère. Dès qu'il n'y plus d'entretien, la compétition s'installe et provoque une diminution du nombre des espèces jusqu'à un minimum $\left(5 \mathrm{esp} . / \mathrm{m}^{2}\right)$. Si le vieillissement de la formation est possible, cas de P4, nous observons l'installation d'espèces de milieux plus stables. Elles ont une longue durée de vie, Cependant ce phénomène de stabilisation est limité en raison de la courte durée du cycle d'exploitation : généralement de 15 à 20 ans. Il est désormais de plus en plus difficile d'observer ce phénomène, d'autant qu'aujourd'hui la sélection de nouveaux clones de peupliers (Beaupré, Dorskamp, Hunnegem...) permet d'accélérer les rotations (13 à 15 ans).

Remerciements : Mes remerciements s'adressent à M. le Professeur G. Durrieu et au Dr. E. Tabacchi pour les commentaires et suggestions qu'ils ont bien voulu apporter au cours de la rédaction de ce manuscrit.

\section{Références}

[1] Pickett S.T.A., White P.S., Natural disturbance and patch dynamics: an introduction, in: Pickett S.T.A., White P.S. 
(éds.), The Ecology of Natural Disturbance and Patch Dynamics, Academic Press, Orlando, 1985, pp. 3-13.

[2] Tilman D., Ressource competition and community structure, Princeton University Press, Princeton, NJ, 1982.

[3] Pimm S.L., The complexity and stability of ecosystems, Nature 307 (1984) 321-326.

[4] Gough L., Grace J.B., Taylor K.L., The relationship between species richness and community biomass: the importance of environmental variables, Oikos 70 (1994) 271-279

[5] Elisseou G.C., Veresoglou D.S., Mamolos A.P., Vegetation productivity and diversity of acid grasslands in northern Greece as influenced by dry winter rainfall and limiting nutrients, Acta Oecol. 16 (1995) 687-702.

[6] Al-Mufti M.M., Sydes C.L., Furness S.B., Grime J.P., Band S.R., A quantitative analysis of shoot phenology and dominance in herbaceous vegetation, J. Ecol. 65 (1977) 759-791.

[7] Grime J.P., Plant Strategies and Vegetation Processes, University of Sheffield, 1979.

[8] Rosenzweig M.L., Abramsky Z., How are diversity and productivity related?, in: Ricklefs R.E., Schulter D. (éds), Species Diversity in Ecological Communities, Historical and Geographical Perspectives, 1993, pp. 52-65.

[9] Abrams P.A., Resource productivity-consumer species diversity: simple models of competition in spatially heterogeneous environment, Ecology 69 (1988) 1418-1433.

[10] Décamps H., Fortuné M., Gazelle F., Pautou G., Historical influence of man on the riparian dynamics of a fluvial landscape, Landscape Ecol. 1 (1988) 163-173.

[11] Pinay G., Fabre A., Vervier Ph., Gazelle F., Control of C. N. P. distribution in soils of riparian forests, Landscape Ecol. 6 (1992) 121-132.

[12] Pearson P., Écologie forestière : la forêt, son climat, son sol, ses arbres, sa faune, Gauthier-Villars, Paris, 1974.

[13] Parfonry A., Production d'un écosystème de qualité. Peupleraies et biodiversité, La forêt Wallone 28 (1996) 19-21.

[14] Hunter M. L. Jr, Wildlife, Forests, and Forestry: Principles of Managing Forests for Biological Diversity, Prentice-Hall, Englewood Cliffs, 1990.

[15] Burton PJ., Balisky A.C., Coward L.P., Cumming S.G., Kneeshaw D.D., The value of managing for biodiversity, Forest. Chron. 68 (1992) 225-237.

[16] Tutin T.G., Heywood V.H., Burges N.A., Moore D.M., Valentine D.H., Walters S.M., Webb D.A., Flora Europaea, 5 vol., Cambridge University Press, 1964-1980.

[17] Husnot M., Muscologia Gallica. Descriptions et figures des mousses de France et des contrées voisines, Amsterdam, 1967.

[18] Shannon C.E., A mathematical theory of communication, The Bell System Technical Journal 27 (1948) 379-423, $623-656$. 1975 .

[19] Pielou E.C., Ecological Diversity, Wiley-Interscience,

[20] Tukey J.W., Exploratory Data Analysis, AddisonWesley, Reading, MA, 1987.
[21] SYSTAT, Statistics. Version 5. 2. Editions Evanston, I. L. Systat, Inc., 1992.

[22] Malanson G.P., Riparian Landscapes, Cambridge University Press, Cambridge, 1993.

[23] Huston M.A., De Angelis D.L., Competition and coexistence: the effects of ressource transport and supply rates, Am. Nat. 144 (1994) 954-977.

[24] Huston M., A general hypothesis of species diversity, Am. Nat. 113 (1979) 81-101.

[25] Mac Intyre S., Lavorel S., Tremont R.M., Plant lifehistory attributes: their relationship to disturbance response in herbaceous vegetation, J. Ecol. 83 (1995) 31-44.

[26] Dessaint F., Chadoeuf R., Barralis G., Nine years' soil seed bank and weed vegetation relationships in an arable field without weed control, J. Appl. Ecol. 34 (1997) 123-130.

[27] Raunkiaer C., The Life-forms of Plants and Statistical Plant Geography, Clarendon Press, Oxford, 1934.

[28] Berthelot A., Typlogie de stations dans les peupleraies cultivées, Rev. For. Fr. 49 (1997) 531-544.

[29] Botineau M., Descubes-Gouilly C., Ghestem A., Contribution à l'étude de la végétation des peupleraies de la moyenne vallée de la Vienne entre Limoges (Haute-Vienne) et Exideuil (Charente), Doc. Phytos. 8 (1984) 233-245.

[30] Halpern C.B., Spies T.A., Plant species diversity in natural and managed forests of the Pacific northwest, Ecol. Appl. 5 (1995) 913-934.

[31] Wilson S.D., Keddy P.A., Measuring diffuse competition along an environmental gradient: results from a shoreline plant community, Am. Nat. 127 (1986) 862-869.

[32] Harriot C.A., Fisher J.H., Hood K.J., Smith M.A., Persistence and colonization of gaps in sown swards of grass and clover under different sward management, Grass Forage Sci. 52 (1997) 156-166.

[33] Odum E.P., Ecology, Holt, Rinehart and Winston, New York, 1963.

[34] Valverde T., Silvertown J., Canopy closure rate and forest structure, Ecology 78 (1997) 1555-1562.

[35] Wheeler B.D., Giller K.E., Species richness of herbaceous fen vegetation in Broadland, Norfolk in relation to the quantity of above-ground plant material, J. Ecol. 70 (1982) 179-200.

[36] Shugart H.H., O’ Neill R.V. (éd.), Editor's comment on paper 12-15, in Systems Ecology, Benchmark Paper in Ecology, Vol. 9., 1979, pp. 167-168.

[37] May R.M., What is the chance that a large complex system will be stable?, Nature 238 (1972) 413-414.

[38] Johnson K.H., Vogt K.A., Clark H.J., Schmitz O.J., Vogt D.J., Biodiversity and the productivity and stability of ecosystems, Tree 11 (1996) 372-377.

[39] Tilman D., Pacala S., The maintenance of species richness in plant communities, in: Ricklefs R. E., Schulter D. (éds), Species Diversity in Ecological Communities. Historical and Geographical Perspectives, 1993, pp. 13-26. 